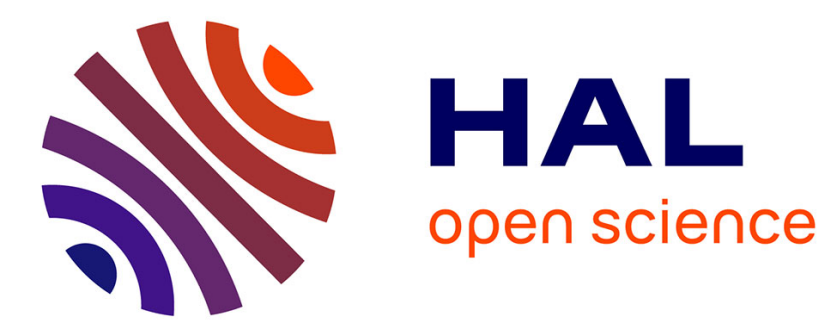

\title{
Cavity Catalysis by Cooperative Vibrational Strong Coupling of Reactant and Solvent Molecules
}

Jyoti Lather, Pooja Bhatt, Anoop Thomas, Thomas Ebbesen, Jino George

\section{To cite this version:}

Jyoti Lather, Pooja Bhatt, Anoop Thomas, Thomas Ebbesen, Jino George. Cavity Catalysis by Cooperative Vibrational Strong Coupling of Reactant and Solvent Molecules. Angewandte Chemie International Edition, 2019, 58 (31), pp.10635-10638. 10.1002/anie.201905407 . hal-03115960

\author{
HAL Id: hal-03115960 \\ https://hal.science/hal-03115960
}

Submitted on 19 Jan 2021

HAL is a multi-disciplinary open access archive for the deposit and dissemination of scientific research documents, whether they are published or not. The documents may come from teaching and research institutions in France or abroad, or from public or private research centers.
L'archive ouverte pluridisciplinaire HAL, est destinée au dépôt et à la diffusion de documents scientifiques de niveau recherche, publiés ou non, émanant des établissements d'enseignement et de recherche français ou étrangers, des laboratoires publics ou privés. 


\section{Cavity Catalysis by Cooperative Vibrational Strong Coupling of Reactant and Solvent Molecules}

Jyoti Lather ${ }^{[1]}$, Pooja Bhatt ${ }^{[1]}$, Anoop Thomas ${ }^{[2]}$, Thomas W. Ebbesen ${ }^{[2] *}$, Jino George ${ }^{[1] *}$

1 Department of Chemical Sciences, Indian Institute of Science Education and Research (IISER) Mohali, Punjab-140306, India. *E-mail: jgeorge@iisermohali.ac.in

2 University of Strasbourg, CNRS, ISIS \& icFRC, 8 allée G. Monge, 67000 Strasbourg, France. *E-mail: ebbesen@unistra.fr

\begin{abstract}
Here we report the catalytic effect of vibrational strong coupling (VSC) on the solvolysis of para-nitrophenyl acetate (PNPA) whereby the reaction rate is increased by an order of magnitude. This is observed when the microfluidic Fabry-Perot cavity, in which the VSC is generated, is tuned to the $C=O$ vibrational stretching mode of both the reactant and solvent molecules.

Thermodynamic experiment confirms the catalytic nature of VSC of the system. The change in the reaction rate follows an exponential relation with respect to the coupling strength of the solvent indicating a cooperative effect between the solvent molecules and the reactant. Furthermore, the study of the solvent kinetic isotope effect clearly shows that the vibrational overlap of $C=O$ vibrational bands of reactant and the strongly coupled solvent molecules is critical to observe the catalysis in this reaction. The combination of cooperative effect and cavity catalysis confirms the potential of VSC as a new frontier in chemistry.
\end{abstract}

Strong light-matter coupling offers a unique way of controlling internal reaction coordinates by coupling molecular transitions to the vacuum field (zero-point energy) of a cavity mode. ${ }^{[1-7]}$ VSC was introduced to couple selectively a vibrational mode, ${ }^{[8,9]}$ which is easily achieved in the liquid state in a microfluidic Fabry-Perot (FP) cavity. ${ }^{[10]}$ Thomas et al., observed the modification of the rate of a C-Si bond breaking process and more recently demonstrated changes to the branching ratio of products by selective VSC of silane derivatives. ${ }^{[3,6]}$ Recently, Hiura et al reported the possibility of accelerating reactions by vibrational ultra-strong coupling of water molecules involved in hydrolysis reactions. ${ }^{[7]}$ In both cases, reactants were directly coupled to cavity modes and the reaction rates could be controlled by moving the physical distance between the mirrors of a FP cavity, i.e. by tuning the coupling to a given vibrational resonance. Recent theoretical studies confirm that potential energy surfaces and reactivity can be modified by coupling to the vacuum field, although the critical role of the solvent is hard to simulate. ${ }^{[11-16]}$

VSC condition can be achieved by injecting a molecular liquid into an FP cavity in which the vibrational transition interacts with the cavity mode (Figure 1a) to generate two new eigen states, called vibro-polaritonic states ( $\mathrm{P}+$ and $\mathrm{P}-)$. The interaction energy (Rabi energy; $\hbar \Omega_{\mathrm{VR}}$ ) is proportional to the transition dipole moment $d$ and the electric field $(E)$ of the electromagnetic mode of the cavity:

$\hbar \Omega_{\mathrm{VR}} \propto 2 d \cdot E \times \sqrt{n_{\mathrm{ph}}+1} ; \quad E=\sqrt{\frac{\hbar \omega}{2 \varepsilon_{0} V}}$

(1)

where $\varepsilon_{0}$ is the vacuum permittivity, $\omega$ is the vibrational frequency, $V$ is the mode volume of the cavity and $n_{\mathrm{ph}}$ the number of photons involved in the coupling process. When the latter goes to zero, a residual Rabi splitting energy remains even in the dark due to the coupling to the zero-point energy of the cavity. Importantly, $\hbar \Omega_{\mathrm{VR}}$ is proportional to $\sqrt{N / V} \sim \sqrt{C}$ where $\mathrm{N}$ is the number and $C$ the concentration of the coupled molecules interacting strongly with the cavity mode. ${ }^{[1-3]}$

In this communication, we report the solvolysis of PNPA catalysed by VSC under cooperative strong coupling effect between the reactant and the solvent molecules. In other words, when the solvent and the reactant molecules have the same vibrational bands but only the solvent is at sufficiently high concentration to be strongly 
coupled to the cavity mode, it increases the reaction rate more than one order of magnitude at room temperature.

(a)

苨

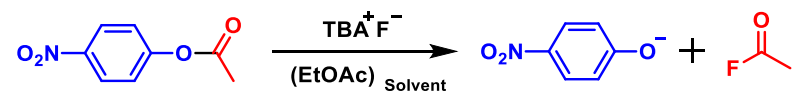

Figure 1. a) Schematic representation of vibropolaritonic states formed from a molecular vibrational state and a F-P cavity mode. b) The hydrolysis of PNPA in ethyl acetate (EtOAc).

The FP cavities used in the present experiments were prepared with Au mirrors sputtered on to IR transparent $\mathrm{BaF}_{2}$ windows by following a reported procedure. ${ }^{[10]}$ The Au mirrors were protected with $100 \mathrm{~nm}$ of sputtered SiOx. The

(a)

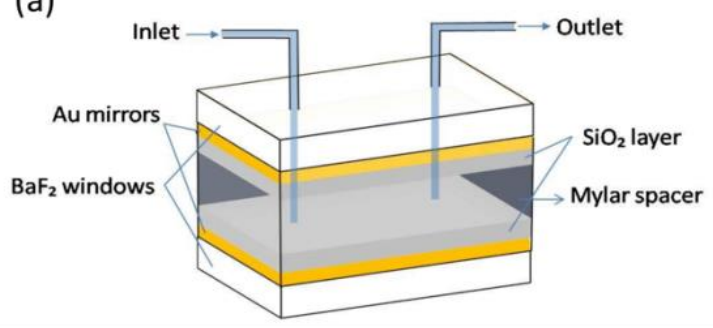

(b)

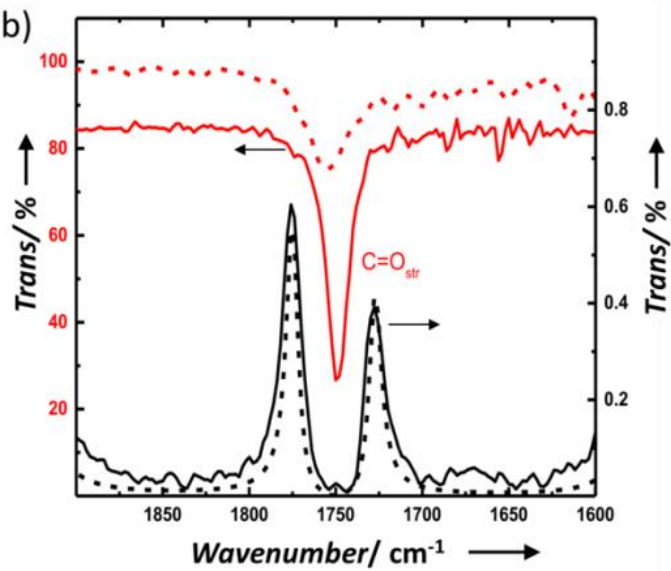

spacing between the mirrors used for the current study is set to $18 \mu \mathrm{m}$ to couple the $\mathrm{C}=\mathrm{O}$ stretching mode of PNPA molecules (Figure 2a). Such F-P cavities can hold up to $3 \mu \mathrm{L}$ volume of reacting solution. The non-cavity experiments were carried out in a microfluidic cell made of the same windows without the Au mirrors. See Supporting Information (SI) for further details. The PNPA solvolysis ${ }^{[17-19]}$ in this study was carried out under mild basic conditions provided by tetrabutylammonium fluoride (TBAF), as schematically shown in Figure 1b. TBAF in Ethyl acetate (EtOAc) normally follows base catalysed acyl ( $B_{A c 2}$ ) bond breaking mechanism (see $S /$ and Scheme S1). EtOAc was chosen as the solvent because its carbonyl stretching transition overlaps with that of the reactant PNPA (Figure $2 \mathrm{~b}$; red traces). Both PNPA and TBAF solutions were prepared separately, mixed outside and injected into the FP cavity.

Figure 2. a) Parts of a flow cell micro cavity-QED reactor. b) IR transmission spectra of $10 \%$ EtOAC (red trace) in hexane and 100 times magnified 0.1 M PNPA (dotted red trace). Vibro-polaritonic states $\mathrm{P}+$ and $\mathrm{P}$ - formed by coupling to the $10^{\text {th }}$ cavity mode (black trace; pathlength is approx. 18 $\mu \mathrm{m})$ with TMM simulation (dotted black trace). Strong coupling of the $\mathrm{C}=\mathrm{O}$ stretching mode of neat ${ }^{12} \mathrm{EtOAc}$ to the $10^{\text {th }}$ mode of the F-P cavity gives a Rabi splitting of $155 \mathrm{~cm}^{-1}$, which is equivalent to $4.4 \%$ change in the actual transition energy with $\mathrm{P}+$ and $\mathrm{P}$ - at $1840 \mathrm{~cm}^{-1}$ and $1685 \mathrm{~cm}^{-1}$, respectively (Figure $2 \mathrm{~b}$ and Figure S2). In the reactive mixture, the PNPA represents only $0.1 \mathrm{w} \%$ of the mixture and is thus unable to undergo strong coupling to the FP cavity at such low concentrations. ${ }^{[1]}$ One of the questions we address here is whether VSC of ${ }^{12} \mathrm{EtOAc}$ can nevertheless influence the reactivity of PNPA in such conditions even though it is not a reactant. The major product of the PNPA solvolysis is the para-nitro phenoxide (PNP-) having an intense absorption peak at $407 \mathrm{~nm}$. Since the transparency region of $\mathrm{BaF}_{2}$ windows extends well in to the UV (ca. $200 \mathrm{~nm}$ ), the progress of the reaction can be easily monitored by following the temporal rise of PNP- absorption peak as shown in Figure 3a (see also Figure S6). In other words, while the FP cavity is set to couple the $\mathrm{C}=\mathrm{O}$ vibrational band in the IR, the influence of strong coupling on the reaction rate is being monitored in the UV-Vis region. This condition helps to avoid complications such as filter effects and other issues 


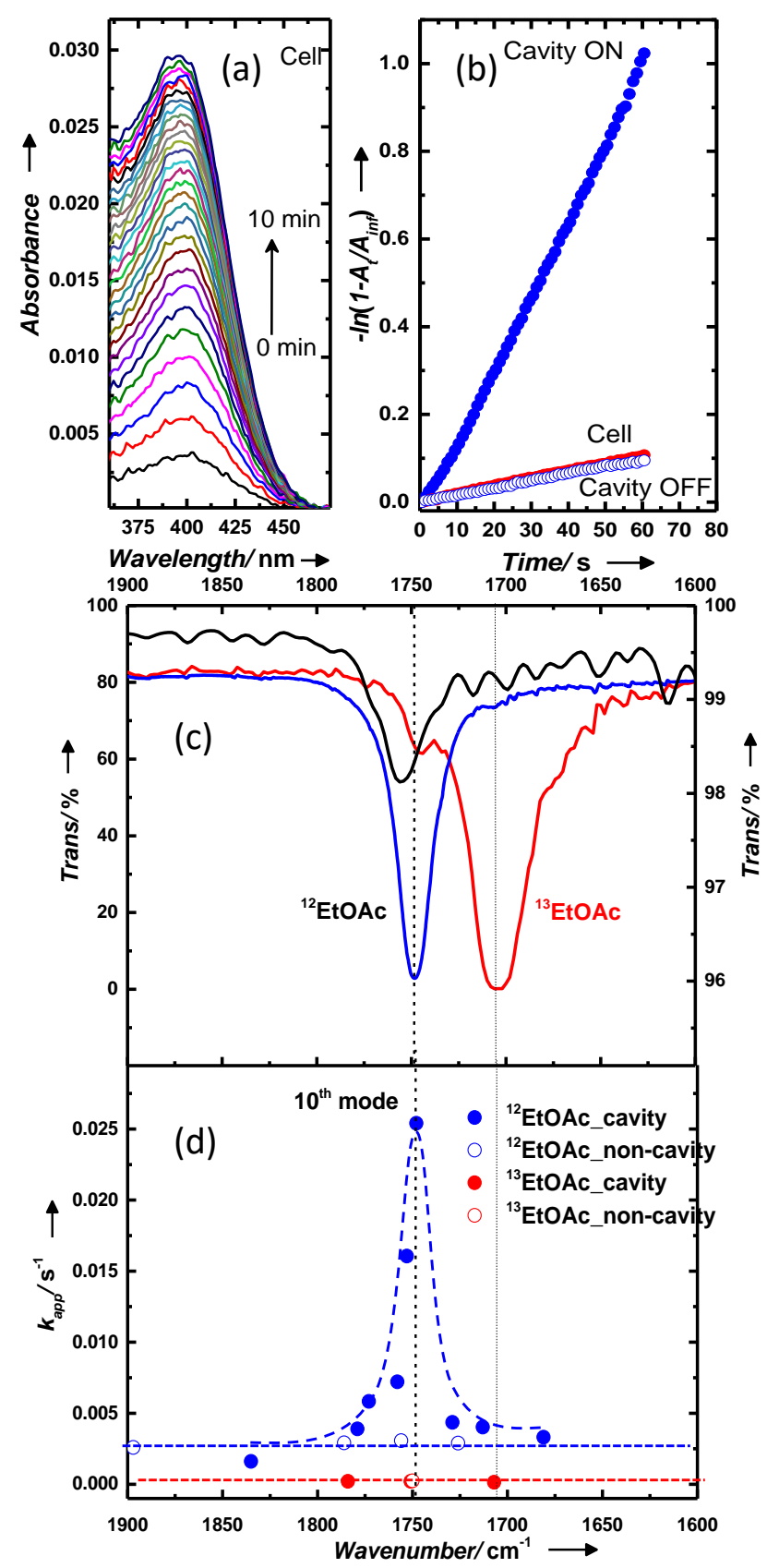

Figure 3. a) Absorption spectra shows the evolution of PNP- species during the ester hydrolysis. b) Pseudo first order kinetic traces measured at $407 \mathrm{~nm}$ for cavity ON-resonance (blue circle; $1.6 \times 10^{-2}$ ), cavity OFF-resonance (blue hollow circle; $0.16 \times 10^{-2}$ ) and non-cavity (red circle; $0.18 \times 10^{-2}$ ) for ${ }^{12}$ EtOAc. c) Carbonyl stretching modes of pure PNPA (ATR spectrum; black trace); FTIR spectra of ${ }^{12} \mathrm{EtOAc}$ (blue trace) and ${ }^{13} \mathrm{EtOAC}$ (red trace) molecules (10\% in hexane). d) The corresponding kinetic traces measured by tuning the cavity $\left({ }^{12}\right.$ EtOAc blue circle; ${ }^{13} \mathrm{EtOAc}$ red circle) and non-cavity ( ${ }^{12} \mathrm{EtOAc}$ blue empty circle; ${ }^{13} \mathrm{EtOAc}$ red empty circle); $10^{\text {th }}$ mode of the cavity overlap with the carbonyl stretching mode of ${ }^{12}$ EtOAc and PNPA. The dashed curves are guides to the eye.

related to the FP cavity configuration. By having a tenfold excess of PNPA as compared to TBAF (0.1 $M$ vs $0.01 \mathrm{M})$, a pseudo-first order kinetics is observed. Apparent rate constants $\left(k_{a p p}\right)$ were determined by linear regression method (Figure $3 b) .{ }^{[17]}$ In the normal non-cavity, the apparent rate constant $\left(\mathrm{kapp}^{\mathrm{nc}}\right)$ is on the order of $0.2 \times 10^{-2}$ $\mathrm{s}^{-1}$ (Figure $3 \mathrm{~b}$ ). To prepare an $\mathrm{ON}$-resonance cavity, the empty cavity is tuned slowly to the desired pathlength and is kept undisturbed for 30 minutes (Supporting Information). Subsequently, the reaction mixture is injected into the cavity and the kinetic traces are recorded. Under ONresonance condition, in other words when the cavity is tuned to ${ }^{12} \mathrm{C}=\mathrm{O}$ stretching mode of ethylacetate ( $\left.{ }^{12} \mathrm{EtOAc}\right)$, the $k_{a p p}{ }^{c a v}$ increased by more than one order of magnitude $\left(2.5 \times 10^{-2} \mathrm{~s}^{-1}\right)$ compared to the non-cavity as shown in Figure 3 b. $k_{a p p}{ }^{c a v}$ levels off to normal values $\left(k_{a p p}{ }^{n c}\right)$ when the cavity is detuned from the $\mathrm{C}=\mathrm{O}$ mode of ${ }^{12} \mathrm{EtOAc}$ (the OFF-resonance measurements) as shown in Figure $3 \mathrm{~d}$. Please note that the noncavity and the cavity conditions are exactly the same, except the presence of Au mirrors in cavity, and $k_{a p p}{ }^{n c}$ is unaffected by small variation in the spacer thickness (blue empty circle in the Figure $3 d)$. Interestingly, the increase in $k_{a p p}{ }^{\text {cav }}$ follows the vibration envelope of $\mathrm{C}=\mathrm{O}$ stretching mode of ${ }^{12}$ EtOAc molecules that is coupled to cavity mode (Figure $3 \mathrm{c}$ and d), following its full width-half maximum (FWHM; $25 \mathrm{~cm}^{-1}$ ). This suggest a cooperative effect between the strongly coupled solvent and the reactant.

In order to confirm this cooperativity effect, we have conducted kinetic isotope effect (KIE) experiments using the ${ }^{12} \mathrm{C}=\mathrm{O}\left({ }^{12} \mathrm{EtOAC}\right)$ and ${ }^{13} \mathrm{C}=\mathrm{O}$ $\left({ }^{13} \mathrm{EtOAc}\right)$ isotopes with carbonyl vibrational resonances at 1750 and $1706 \mathrm{~cm}^{-1}$, respectively (Figure 3c). Outside the cavity, the use of ${ }^{13} \mathrm{EtOAc}$ gives an apparent rate one order of magnitude lower $\left({ }^{13} k_{a p p}=2.4 \times 10^{-4} \mathrm{~s}^{-1}\right)$ with respect to the rate with the ${ }^{12} \mathrm{EtOAc}$ isotope. This large change in the reaction rate can be due to a secondary isotope effect in which the ${ }^{12}$ EtOAc is coupled to PNPA carbonyl vibrational state, facilitating external vibrational energy transfer in the system. ${ }^{[20]}$ Please note that the rate determining 
step for the $B_{A c 2}$ mechanism is the attack of nucleophile on the electron deficient carbonyl carbon atom. ${ }^{[17-19]}$ Very interestingly, under VSC of ${ }^{13} \mathrm{EtOAc}$, the reaction rates are not modified.

Kinetic action spectra measured as a function of cavity tuning, Figure $3 d$, shows no change in the rate for the ${ }^{13} \mathrm{EtOAc}$ solvent system relative to rate outside the cavity, in contrast to ${ }^{12} \mathrm{EtOAc}$. This is a confirmation that the VSC of ${ }^{12} \mathrm{EtOAC}$ is acting cooperatively and perturbing the PNPA internal reaction coordinate so as to affect its bond dissociation energy as will be discussed again further down in agreement with recent theoretical predictions. ${ }^{\text {[21] }}$

To clarify how the VSC is affecting the reaction barrier and whether it is truly catalysing the reaction, the temperature dependence of the reaction rate was analysed to extract the thermodynamics activation parameters. As it is immediately apparent from the slopes in Figure $4 a$, the activation energy drops under VSC. The enthalpy of activation $\left(\Delta \mathrm{H}^{\ddagger}\right)$ calculated from Eyring equation decreases substantially from 53.2 $\mathrm{kJ} / \mathrm{mol}$ to $7.9 \mathrm{~kJ} / \mathrm{mol}$ (Figure 4a; Supporting Information), indicating that the transition state (TS) is far more stable under VSC of ${ }^{12}$ EtOAC molecules. Another very interesting finding is that the entropy of activation $\left(\Delta S^{\ddagger}\right)$ also decreases significantly from $-70.9 \mathrm{~J} / \mathrm{K} / \mathrm{mol}$ to $-206.2 \mathrm{~J} / \mathrm{K} / \mathrm{mol}$ offsetting partially the change in $\Delta \mathrm{H}^{\ddagger}$. This large decrease in $\Delta S^{\ddagger}$ indicates that the TS is far more polar under VSC thereby structuring the solvent cage which also decreases $\Delta H^{\ddagger}$. In other words the solvent-solute interactions are much stronger under VSC, thereby favouring $\mathrm{B}_{\mathrm{Ac} 2}$ mechanism by stabilizing the intermediates.
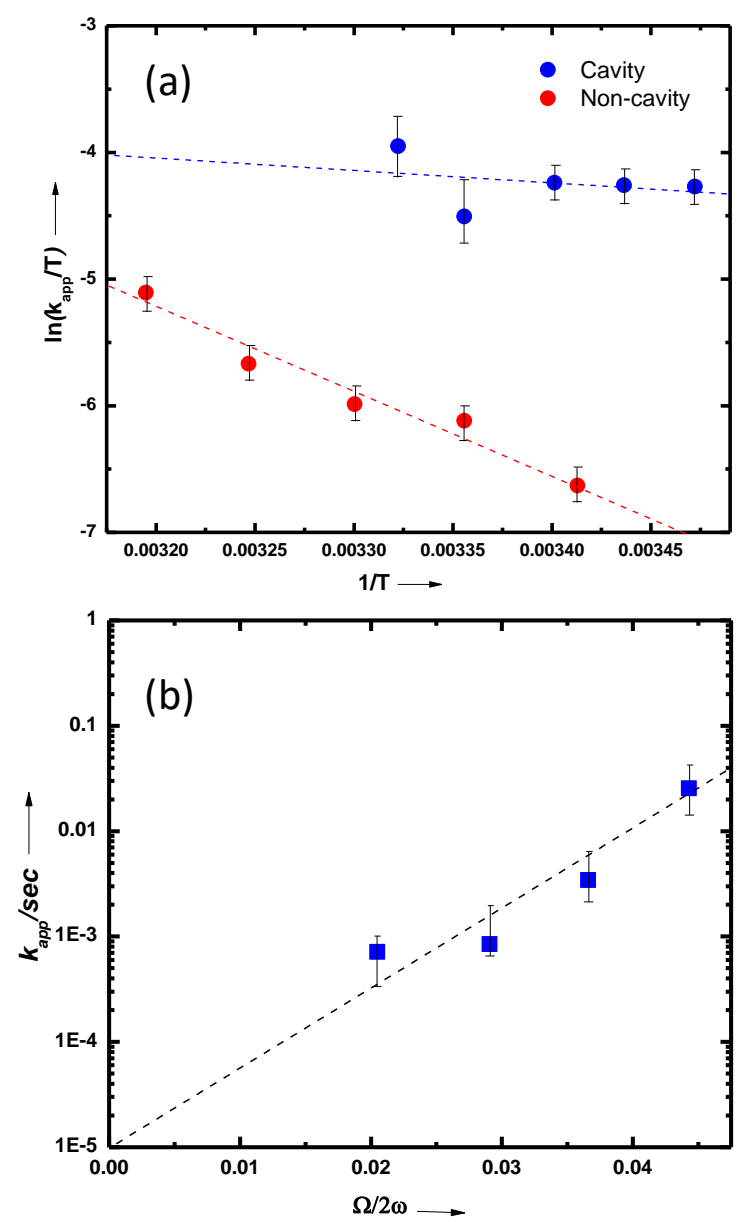

Figure 4. (a) Eyring plot for reaction inside the cavity (blue circles) and non-cavity (cell; red circles); (b) Apparent rate constant as a function of Rabi splitting under VSC of ${ }^{12}$ EtOAc. Dotted lines are the corresponding linear fitting.

We then looked into the reaction rate variation with respect to cooperative coupling strength of the ${ }^{12}$ EtOAc molecules. To do this we fixed the concentration of PNPA (0.1 M) while varying the ${ }^{12}$ EtOAc concentration in a mixed anisole -

${ }^{12}$ EtOAc solution. We chose anisole as a cosolvent; the relative solvent polarity difference is only 0.03 compared to EtOAc and the rate is essentially the same in anisole and ${ }^{12} \mathrm{EtOAc}$ in the absence of VSC (Figure S3) ( ${ }^{12}$ EtOAc to reactant ratio is close to 1000 , we assume that the vibrational bath is still preserved at the lower concentration of ${ }^{12} \mathrm{EtOAc}$ ). An exponential rise in the reaction rate is observed upon increasing the concentration of ${ }^{12}$ EtOAc under strong coupling condition, in other words with the Rabi splitting (Figure 4b). Such an exponential dependence has also been observed for both a slowing desilanation reaction ${ }^{[3]}$ and for an accelerating hydrolysis reaction. ${ }^{[7]}$ This probably points to a 
more general feature that the activation energy varies linearly with the Rabi splitting in whichever direction the barrier is moving under VSC. In conclusion, the thermodynamic data shows unequivocally that the reaction is catalyzed under VSC of the solvent. The large decrease in the entropy of activation indicates that the electron density distribution is strongly modified in the TS under VSC resulting in a more polar activated complex. The solvent concentration dependence and the kinetic isotope effect demonstrates that VSC can be transferred from the solvent to the solute as long as their vibrational resonances overlap. This spectral overlap is not in itself enough to transfer VSC from the solvent to the reactant. Clearly the solute-solvent interactions must be strong, e.g. dipolar coupling, to induce the transfer, as has recently been shown in a theoretical study of ensemble induced strong coupling effects on single quantum objects. ${ }^{[21]}$ Other theoretical studies have considered chemical reactivity under VSC in the gas phase and confirm that the reactivity should be modified. ${ }^{[13-16]}$ In view of the important role of solvation in the TS, further theoretical studies taking into account solvation effects under VSC would be very helpful. On the experimental side, many more classes of reactions need to be studied in order to extract general principles that govern reactions under VSC. The use of cooperative effects as demonstrated here should greatly facilitate this task since it removes the requirement of high concentration of reactants to achieve the strong coupling condition. Action spectra under VSC can be used as a spectroscopic tool to help to elucidate which vibrations play a role in the reaction path. These findings should have large impact on controlling and understanding chemical reactivity through VSC.

\section{Acknowledgements}

J.G. would like to thank Department of Chemical Sciences, IISER Mohali for using the facilities; DSTSERB, Core Research Grant (EMR/2017/003455) is also acknowledged. J. L. and P. B. thank IISER Mohali for research funding. TWE acknowledges support of the International Center for Frontier Research in Chemistry (icFRC, Strasbourg), the ANR Equipex Union (ANR-10-EQPX-52-01), the Labex NIE projects (ANR-11-LABX-0058 NIE) and CSC (ANR-10-LABX- 0026 CSC) within the Investissement d'Avenir program ANR-10-IDEX0002-02 and the ERC (project no 788482 MOLUSC).

Keywords: Cavity Catalysis • Cooperativity • Vibrational Strong Coupling • Polaritonic Chemistry • 
[1] T. W. Ebbesen, Acc. Chem. Res. 2016, 49, 2403-2412.

[2] J. A. Hutchison, T. Schwartz, C. Genet, E. Devaux, T. W. Ebbesen, Angew. Chem. Int. Ed. 2012, 51, 1592-1596.

[3] A. Thomas, J. George, A. Shalabney, M. Dryzhakov, S. J. Varma, J. Moran, T. Chervy, X. Zhong, E. Devaux, C. Genet, Angew. Chem. Int. Ed. 2016, 55, 11462-11466.

[4] B. Munkhbat, M. Wersäll, D. G. Baranov, T. J. Antosiewicz, T. Shegai, Sci. Adv. 2018, 4, eaas9552.

[5] V. N. Peters, M. O. Faruk, J. Asane, R. Alexander, D. A. Peters, S. Prayakarao, S. Rout, M. A. Noginov, Optica, 2019, 6, 318-325.

[6] A. Thomas, L. Lethuillier-Karl, K. Nagarajan, R. M. Vergauwe, J. George, T. Chervy, A. Shalabney, E. Devaux, C. Genet, J. Moran, Science 2019, 363, 615-619.

[7] H. Hiura, A. Shalabney, J. George, 2018, DOI 10.26434/chemrxiv.7234721.v3.

[8] A. Shalabney, J. George, J. Hutchison, G. Pupillo, C. Genet, T. W. Ebbesen, Nat. Commun. 2015, 6, 5981.

[9] J. P. Long, B. S. Simpkins, ACS Photonics 2015, 2, 130-136.

[10] J. George, A. Shalabney, J. A. Hutchison, C. Genet, T. W. Ebbesen, J. Phys. Chem. Lett. 2015, 6, 1027-1031.

[11] J. Flick, M. Ruggenthaler, H. Appel, A. Rubio, Pro. Nat. Acad. Sci. 2017, 114, 3026-3034. [12] M. Ruggenthaler, N. Tancogne-Dejean, J. Flick, H. Appel, A. Rubio, Nat. Rev. Chem. 2018, 2, 0118.

[13] M. Du, R. F. Ribeiro, J. Yuen-Zhou, Chem 2019, DOI 10.1016/j.chempr.2019.02.009.

[14] J. C. G. Angulo, R. F. Ribeiro, J. Yuen-Zhou, 2019, arXiv:1902.10264 [cond-mat, physics:physics, physics:quant-ph].

[12] F. Herrera, F. C. Spano, Phys. Rev. Lett. 2016, 116, 238301.

[13] F. Herrera, F. C. Spano, ACS Photonics 2018, 5, 65-79.

[14] J. Galego, F. J. Garcia-Vidal, J. Feist, Nat. Commun. 2016, 7, 13841.

[15] C. Climent, J. Galego, F. J. Garcia-Vidal, J. Feist, Angew. Chem. Int. Ed. 2019, 0, DOI 10.1002/anie.201901926.
[16] J. Galego, C. Climent, F. J. Garcia-Vidal, J. Feist, 2018, arXiv:1807.10846 [physics, physics:quant-ph].

[17] J. H. Park, B. P. Meriwether, P. Clodfelder, L. W. Cunningham, J. Bio. Chem. 1961, 236, 136141.

[18] C. Mitton, R. Schowen, M. Gresser, J.

Shapley, J. Am. Chem. Soc. 1969, 91, 2036-2044.

[19] C. G. Mitton, M. Gresser, R. L. Schowen, J. Am. Chem. Soc. 1969, 91, 2045-2047.

[20] J.C. Owrutsky, A. D. Raftery, R. M. Hochstrasser, Ann. Rev. Phys. Chem. 1994, 45, 519-555.

[21] S. Schütz, J. Schachenmayer, D. Hagenmüller, V. Sandoghdar, T. W. Ebbesen, C. Genes, G. Pupillo, 2019, arXiv:1904.08888 [quantph 
